\title{
Analysis of TCP-AQM Interaction via Periodic Optimization and Linear Programming: The Case of Sigmoidal Utility Function *
}

\author{
K. Avrachenkov ${ }^{1}$, L. Finlay ${ }^{2}$, and V. Gaitsgory ${ }^{2}$ \\ 1 INRIA Sophia Antipolis, France \\ K. Avrachenkov@sophia.inria.fr \\ 2 University of South Australia, Australia \\ \{luke.finlay, vladimir.gaitsgory\}@unisa.edu.au
}

\begin{abstract}
We investigate the interaction between Transmission Control Protocol (TCP) and an Active Queue Management (AQM) router, that are designed to control congestion in the Internet. TCP controls the sending rate with which the data is injected into the network and AQM generates control signals based on the congestion level. For a given TCP version, we define the optimal strategy for the AQM router as a solution of a nonlinear periodic optimization problem, and we find this solution using a linear programming approach. We show that depending on the choice of the utility function for the sending rate, the optimal control is either periodic or steady state. Main attention is paid to a problem with a sigmoidal utility function, in which the evolution of the optimal sending rate resembles a "saw-tooth" behavior of the "instantaneous" TCP sending rate.
\end{abstract}

\section{Introduction}

Most traffic in the Internet is governed by the TCP/IP protocol [3], [10]. Data packets of an Internet connection travel from a source node to a destination node via a series of routers. Some routers, particularly edge routers, experience periods of congestion when packets spend a non-negligible time waiting in the router buffers to be transmitted over the next hop. The TCP protocol tries to adjust the sending rate of a source to match the available bandwidth along the path. During the principle Congestion Avoidance phase the current TCP New Reno version uses Additive Increase Multiplicative Decrease (AIMD) binary feedback congestion control scheme. In the absence of congestion signals from the network TCP increases sending rate linearly in time, and upon the reception of a congestion signal TCP reduces the sending rate by a multiplicative factor. Thus, the instantaneous AIMD TCP sending rate exhibits a "saw-tooth"

\footnotetext{
* The work was supported by the Australian Research Council Discovery-Project Grants DP0346099, DP0664330, Linkage International Grant LX0560049 and France Telecom R\&D Grant "Modélisation et Gestion du Trafic réseaux Internet" no. 42937433.
} 
behavior. Congestion signals can be either packet losses or Explicit Congestion Notifications (ECN) [17]. At the present state of the Internet, nearly all congestion signals are generated by packet losses. Packets can be dropped either when the router buffer is full or when an Active Queue Management (AQM) scheme is employed [7]. In particular, AQM RED [7] drops or marks packets with a probability which is a piece-wise linear function of the average queue length. Given an ambiguity in the choice of the AQM parameters (see [4] and [15]), so far AQM is rarely used in practice. In the present work, we study the interaction between TCP and AQM. In particular, we pose and try to answer the question: What should be the optimal dropping or marking strategy in the AQM router? For the performance criterion, we choose the average utility function of the throughput minus either the average cost of queueing or the average cost of losses. This performance criterion with a linear utility function was introduced in [1]. We have analyzed not only the currently used AIMD congestion control, but also Multiplicative Increase Multiplicative Decrease (MIMD) congestion control. In particular, MIMD (or Scalable TCP [11]) is proposed for congestion control in high speed networks. However, since it turns out that the results for MIMD and AIMD congestion control schemes are similar, we provide the detailed analysis only for AIMD TCP.

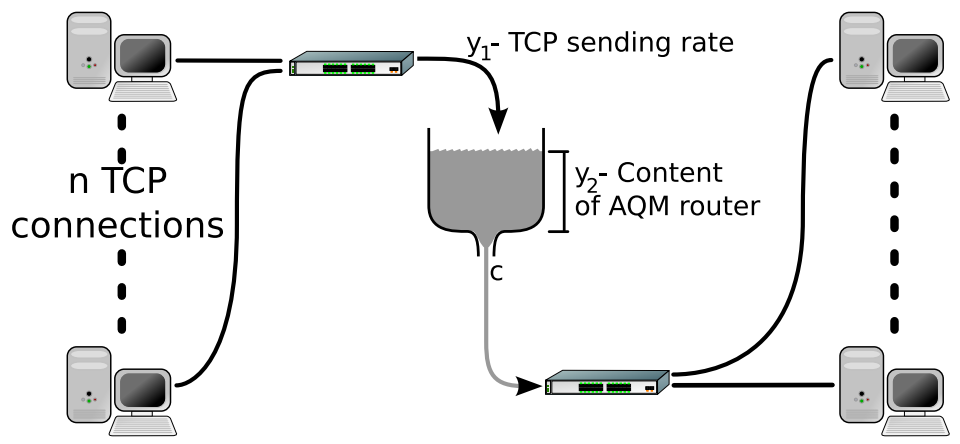

Fig. 1. Fluid model for data network.

We restrict the analysis to the single bottleneck network topology (see Figure 1). In particular, we suppose that $n$ TCP connections cross a single bottleneck router with the AQM mechanism. We take the fluid approach for modeling the interaction between TCP and AQM [13], [14], [19]. In such an approach, the variables stand for approximations of average values and their evolution is described by deterministic differential equations. Since we consider long-run time average criteria, our TCP-AQM interaction model falls into the framework of the periodic optimization (as outlined in the next section). 
This paper is an updated version of the earlier authors' work [2]. The main difference from [2] is that in the current paper we consider (and present numerical results for) a practically important case of a sigmoidal utility function.

\section{Statement of the problem}

Consider the control system

$$
\dot{y}(t)=f(u(t), y(t)), \quad t \in[0, T], \quad T>0,
$$

where the function $f(u, y): U \times \mathbb{R}^{m} \rightarrow \mathbb{R}^{m}$ is continuous in $(u, y)$ and satisfies Lipschitz conditions in $y$; the controls are Lebesque measurable functions $u(t)$ : $[0, T] \rightarrow U$ and $U$ is a compact subset of $\mathbb{R}^{n}$.

Let $Y$ be a compact subset of $\mathbb{R}^{m}$. A pair $(u(t), y(t))$ will be called admissible on the interval $[0, T]$ if the equation (1) is satisfied for almost all $t \in[0, T]$ and $y(t) \in Y \quad \forall t \in[0, T]$. A pair $(u(t), y(t))$ will be called periodic admissible on the interval $[0, T]$ if it is admissible on $[0, T]$ and $y(0)=y(T)$.

Let $g(u, y): U \times \mathbb{R}^{m} \rightarrow \mathbb{R}^{1}$ be a continuous function. The following problem is commonly referred to as the periodic optimization problem:

$$
\sup _{(u(\cdot), y(\cdot))} \frac{1}{T} \int_{0}^{T} g(u(t), y(t)) d t \stackrel{\text { def }}{=} G_{p e r}
$$

where sup is over the length of the time interval $T>0$ and over the periodic admissible pairs defined on $[0, T]$.

A very special family of periodic admissible pairs is that consisting of constant valued controls and corresponding steady state solutions of (1):

$$
(u(t), y(t))=(u, y) \in \mathcal{M} \stackrel{\text { def }}{=}\{(u, y) \mid(u, y) \in U \times Y, f(u, y)=0\} .
$$

If sup is sought over the admissible pairs from this family, the problem (2) is reduced to

$$
\sup _{(u, y) \in \mathcal{M}} g(u, y) \stackrel{\text { def }}{=} G_{s s}
$$

which is called a steady state optimization problem. Note that

$$
G_{p e r} \geq G_{s s}
$$

and that, as can be easily verified, $G_{p e r}=G_{s s}$ if the system (1) is linear, the sets $U, Y$ are convex and the function $g(u, y)$ is concave. Note also that in a general case (e.g., the dynamics is non-linear and/or the integrand is not concave), (5) can take the form of a strict inequality (examples can be found in $[5],[8],[9]$ and in references therein).

We formulate the problem of optimal control of TCP-AQM interaction as a periodic optimization problem, in which the state space is two dimensional

$$
y=\left(y_{1}, y_{2}\right), \quad f(u, y)=\left(f_{1}\left(u, y_{1}\right), f_{2}\left(u, y_{1}\right)\right) ;
$$


and the control $u$ is a scalar: $u(t) \in U$, with

$$
U \stackrel{\text { def }}{=}\{u: 0 \leq u \leq 1\} .
$$

We consider two congestion control schemes: Additive Increase Multiplicative Decrease (AIMD) scheme and Multiplicative Increase Multiplicative Decrease (MIMD) scheme. In both cases the first state component $y_{1}(t)$ is interpreted as a sending rate at the moment $t$, while the second state component $y_{2}(t)$ represents the size of the queue in the router buffer. In the AIMD scheme, the evolution of $y_{1}(t)$ is defined by the equation

$$
\dot{y}_{1}(t)=f_{1}\left(u(t), y_{1}(t)\right) \stackrel{\text { def }}{=} \alpha(1-u(t))-\beta y_{1}^{2}(t) u(t),
$$

where $\alpha=\alpha_{0} n / \tau^{2}$ and $\beta=1-\beta_{0} / n$. Here $n$ is the number of competing TCP connections, $\tau$ is the round trip time. Typical values for $\alpha_{0}$ and $\beta_{0}$ are 1 and 0.5 , respectively. In the MIMD scheme, the evolution of $y_{1}(t)$ is defined by the equation

$$
\dot{y}_{1}(t)=f_{1}\left(u(t), y_{1}(t)\right) \stackrel{\text { def }}{=} \gamma y_{1}(t)(1-u(t))-\beta y_{1}^{2}(t) u(t),
$$

where $\gamma=\gamma_{0} / \tau$ and $\beta$ as in the AIMD case. A typical value for $\gamma_{0}$ is 0.01 . The control $u(t)$ is interpreted as the dropping/marking probability. A detail derivation of equation (8) can be found for instance in [13] and [19]. Note also that if the control is not applied $(u(t)=0)$, the sending rate grows linearly in time if AIMD is used, and the sending rate grows exponentially in time if MIMD is used.

We study active queue management with and without explicit congestion notifications. When AQM with ECN is used, the packets are not dropped from the buffer when control is applied and the buffer is not full. In the case of AQM with ECN (AQM-ECN scheme), the evolution of the router buffer content $y_{2}(t)$ is described by

$$
\dot{y}_{2}(t)=f_{2}\left(u(t), y_{1}(t)\right)=f_{2}\left(y_{1}(t)\right) \stackrel{\text { def }}{=}\left\{\begin{array}{l}
y_{1}(t)-c, \quad 0<y_{2}(t)<B, \\
{\left[y_{1}(t)-c\right]_{+}, y_{2}(t)=0,} \\
{\left[y_{1}(t)-c\right]_{-}, y_{2}(t)=B,}
\end{array}\right.
$$

where $c$ is the router capacity, $B$ is the buffer size, $[a]_{+}=\max (a, 0)$ and $[a]_{-}=$ $\min (a, 0)$. In the case of AQM without ECN (AQM-non-ECN scheme), AQM signals congestion by dropping packets with rate $u(t) y_{1}(t)$. Consequently, the dynamics of the router buffer content $y_{2}(t)$ is described by

$$
\dot{y}_{2}(t)=f_{2}\left(u(t), y_{1}(t)\right) \stackrel{\text { def } f}{=}\left\{\begin{array}{l}
(1-u(t)) y_{1}(t)-c, \quad 0<y_{2}(t)<B, \\
{\left[(1-u(t)) y_{1}(t)-c\right]_{+}, y_{2}(t)=0,} \\
{\left[(1-u(t)) y_{1}(t)-c\right]_{-}, y_{2}(t)=B .}
\end{array}\right.
$$

The function $g(u, y)$ in the objective (2) will be defined as follows

$$
g(u, y)=\psi\left(y_{1}\right)-\kappa y_{2}-M e^{K\left(y_{2}-B\right)} y_{1},
$$


where $\psi(\cdot)$ is the utility function for the sending rate value, $\kappa y_{2}$ is the cost of delaying the data in the buffer, and $M e^{K\left(y_{2}-B\right)} y_{1}$ is the penalty function for losing data when the buffer is full. Examples of the utility functions we will be dealing with are:

$$
\psi\left(y_{1}\right)=y_{1}, \quad \psi\left(y_{1}\right)=\log \left(1+y_{1}\right), \quad \psi\left(y_{1}\right)=\frac{y_{1}^{2}}{y_{1}^{2}+a}
$$

The linear utility function corresponds to the throughput maximization. The concave utility function such as logarithm is the conventional utility function for elastic applications in the Internet. Finally, the sigmoidal utility function corresponds well to the audio and video streaming applications $[6,12,18]$. The sigmoidal utility function has a single inflexion point which separates a convex part for low sending rates and a concave part for high sending rates.

The rest of the paper is organized as follows: In Section 3 we give an overview of the linear programming approach to periodic optimization. Then, in Sections 4 and 5 we apply the general technique of Section 3 to the problem of interaction between TCP and AQM. We show that depending on the utility function for the sending rate, we obtain either periodic or steady state optimal solution. We conclude the paper with Section 6.

\section{Linear programming approach}

In [9] it has been shown that the periodic optimization problem (2) can be approximated by a family of finite dimensional Linear Programming Problems (LPPs) (called in the sequel as approximating LPP). This approximating LPP is constructed as follows.

Let $y_{j}(j=1, \ldots, m)$ stand for the $j$ th component of $y$ and let $\phi_{i}(y)$ be the monomomial:

$$
\phi_{i}(y) \stackrel{\text { def }}{=} y_{1}^{i_{1}} \ldots y_{m}^{i_{m}}
$$

where $i$ is the multi-index: $i \stackrel{\text { def }}{=}\left(i_{1}, \ldots, i_{m}\right)$. Let us denote by $I_{N}$ the set of multi-indices

$$
I_{N} \stackrel{\text { def }}{=}\left\{i: i=\left(i_{1}, \ldots, i_{m}\right), \quad i_{1}, \ldots, i_{m}=0,1, \ldots, N, \quad i_{1}+\ldots+i_{m} \geq 1\right\} .
$$

Note that the number of elements in $I_{N}$ is $(N+1)^{m}-1$. Assume that, for any $\Delta>0$, the points $\left(u_{l}^{\Delta}, y_{k}^{\Delta}\right) \in U \times Y, l=1, \ldots, L^{\Delta}, k=1, \ldots, K^{\Delta}$, are being chosen in such a way that, for any $(u, y) \in U \times Y$, there exists $\left(u_{l}^{\Delta}, y_{k}^{\Delta}\right)$ such that $\left\|(u, y)-\left(u_{l}^{\Delta}, y_{k}^{\Delta}\right)\right\| \leq c \Delta$, where $c$ is a constant.

Define the polyhedral set $W_{N}^{\Delta} \subset \mathbb{R}^{L^{\Delta}+K^{\Delta}}$

$$
W_{N}^{\Delta} \stackrel{\text { def }}{=}\left\{\gamma=\left\{\gamma_{l, k}\right\} \geq 0: \sum_{l, k} \gamma_{l, k}=1\right.
$$




$$
\left.\sum_{l, k}\left(\phi_{i}^{\prime}\left(y_{k}^{\Delta}\right)\right)^{T} f\left(u_{l}^{\Delta}, y_{k}^{\Delta}\right) \gamma_{l, k}=0, i \in I_{N}\right\},
$$

where $\phi_{i}^{\prime}(\cdot)$ is the gradient of $\phi_{i}(\cdot)$. Define the approximating LPP as follows

$$
\max _{\gamma \in W_{N}^{\Delta}} \sum_{l, k} \gamma_{l, k} g\left(u_{l}^{\Delta}, y_{k}^{\Delta}\right) \stackrel{\text { def }}{=} G_{N}^{\Delta}
$$

where $\sum_{l, k} \stackrel{\text { def }}{=} \sum_{l=1}^{L^{\Delta}} \sum_{k=1}^{K^{\Delta}}$.

As shown in [9] (under certain natural and easily verifiable conditions), there exists the limit of the optimal value $G^{N, \Delta}$ of the LPP (15) and this limit is equal to the optimal value $G_{p e r}$ of the periodic optimization problem (2):

$$
\lim _{N \rightarrow \infty} \lim _{\Delta \rightarrow 0} G_{N}^{\Delta}=G_{p e r} .
$$

Also, for any fixed $N$,

$$
\lim _{\Delta \rightarrow 0} G_{N}^{\Delta} \stackrel{\text { def }}{=} G_{N} \geq G_{p e r} .
$$

Thus, $G_{N}^{\Delta}$ can be used as an approximation of $G_{p e r}$ if $N$ is large and $\Delta$ is small enough.

Let $\left(u^{*}(\cdot), y^{*}(\cdot)\right)$ be the solution of the periodic optimization problem (2) defined on the optimal period $T=T^{*}$ (assuming that this solution exists and is unique) and let $\gamma^{N, \Delta} \stackrel{\text { def }}{=}\left\{\gamma_{l, k}^{N, \Delta}\right\}$ be an optimal basic solution of the approximating LPP (15). From the consideration in [9] it follows that an element $\gamma_{l, k}^{N, \Delta}$ of $\gamma^{N, \Delta}$ can be interpreted as an estimate of the "proportion" of time spent by the optimal pair $\left(u^{*}(\cdot), y^{*}(\cdot)\right)$ in a $\Delta$-neighborhood of the point $\left(u_{l}, y_{k}\right)$, and in particular, the fact that $\gamma_{l, k}^{\Delta}$ is positive or zero can be interpreted as an indication of that whether or not the optimal pair attends the $\Delta$-neighborhood of $\left(u_{l}, y_{k}\right)$.

Define the set $\Theta$ by the equation

$$
\Theta \stackrel{\text { def }}{=}\left\{(u, y):(u, y)=\left(u^{*}(\tau), y^{*}(\tau)\right) \text { for some } \tau \in\left[0, T^{*}\right]\right\} .
$$

This $\Theta$ is the graph of the optimal feedback control function, which is defined on the optimal state trajectory $\mathcal{Y} \stackrel{\text { def }}{=}\{y:(u, y) \in \Theta\}$ by the equation $\psi(y) \stackrel{\text { def }}{=} u \forall(u, y) \in \Theta$. For the definition of $\psi(\cdot)$ to make sense, it is assumed that the set $\Theta$ is such that from the fact that $\left(u^{\prime}, y\right) \in \Theta$ and $\left(u^{\prime \prime}, y\right) \in \Theta$ it follows that $u^{\prime}=u^{\prime \prime}$ (this assumption being satisfied if the closed curve defined by $y^{*}(\tau), \tau \in\left[0, T^{*}\right]$ does not intersect itself).

Define also the sets:

$$
\begin{gathered}
\Theta_{N}^{\Delta} \stackrel{\text { def }}{=}\left\{\left(u_{l}^{\Delta}, y_{k}^{\Delta}\right): \gamma_{l, k}^{N, \Delta}>0\right\}, \\
\mathcal{Y}_{N}^{\Delta} \stackrel{\text { def }}{=}\left\{y:(u, y) \in \Theta_{N}^{\Delta}\right\}, \\
\psi_{N}^{\Delta}(y) \stackrel{\text { def }}{=} u \forall(u, y) \in \Theta_{N}^{\Delta},
\end{gathered}
$$


where again it is assumed that from the fact that $\left(u^{\prime}, y\right) \in \Theta_{N}^{\Delta}$ and $\left(u^{\prime \prime}, y\right) \in \Theta_{N}^{\Delta}$ it follows that $u^{\prime}=u^{\prime \prime}$. Note that the set $\Theta_{N}^{\Delta}$ (and the set $\mathcal{Y}_{N}^{\Delta}$ ) can contain no more than $(N+1)^{m}$ elements since $\gamma^{N, \Delta}$, being a basic solution of the LPP (15), has no more than $(N+1)^{m}$ positive elements (the number of the equality type constraints in (15)).

As mentioned above, the fact that $\gamma_{l, k}^{N, \Delta}$ is positive or zero can be interpreted as an indication of that whether or not the optimal pair attends the $\Delta$-neighborhood of $\left(u_{l}^{\Delta}, y_{k}^{\Delta}\right)$, and thus, one may expect that $\Theta_{N}^{\Delta}$ can provide some approximation for $\Theta$ if $N$ is large and $\Delta$ is small enough. Such an approximation has been formalized in [9], where it has been established that:

(i) Corresponding to an arbitrary small $r>0$, there exists $N_{0}$ such that, for $N \geq N_{0}$ and $\Delta \leq \Delta_{N}\left(\Delta_{N}\right.$ is positive and small enough),

$$
\Theta \subset \Theta_{N}^{\Delta}+r \mathcal{B}
$$

(ii) Corresponding to an arbitrary small $r>0$ and arbitrary small $\delta>0$, there exists $N_{0}$ such that, for $N \geq N_{0}$ and $\Delta \leq \Delta_{N}\left(\Delta_{N}\right.$ being positive and small enough),

$$
\Theta_{N}^{\Delta, \delta} \subset \Theta+r \mathcal{B}
$$

where $\Theta_{N}^{\Delta, \delta} \stackrel{\text { def }}{=}\left\{\left(u_{l}^{\Delta}, y_{k}^{\Delta}\right): \gamma_{l, k}^{\Delta} \geq \delta\right\}$.

Note that in both $(22)$ and $(23), \mathcal{B}$ is the closed unit ball in $\mathbb{R}^{n+m}$.

The fact that $\Theta_{N}^{\Delta}$ "approximates" $\Theta$ for $N$ large and $\Delta$ small enough leads to the fact that $\mathcal{Y}_{N}^{\Delta}$ approximates $\mathcal{Y}$ and to the fact that $\psi_{N}^{\Delta}(y)$ approximates (in a certain sense) $\psi(y)$. This gives rise to the following algorithm for construction of near-optimal periodic admissible pair [9]:

1) Find an optimal basic solution $\gamma_{N}^{\Delta}$ and the optimal value $G_{N}^{\Delta}$ of the approximating LPP (15) for $N$ large and $\Delta$ small enough; the expression " $N$ large and $\Delta$ small enough" is understood in the sense that a further increment of $N$ and/or a decrement of $\Delta$ lead only to insignificant changes of the optimal value $G_{N}^{\Delta}$ and, thus, the latter can be considered to be approximately equal to $G_{p e r}$ (see $(16))$.

2) Define $\Theta_{N}^{\Delta}, \mathcal{Y}_{N}^{\Delta}, \psi_{N}^{\Delta}(y)$ as in (19). By (22) and (23), the points of $\mathcal{Y}_{N}^{\Delta}$ will be concentrated around a closed curve being the optimal periodic state trajectory while $\psi_{N}^{\Delta}(y)$ will give a point wise approximation to the optimal feedback control.

3) Extrapolate the definition of the function $\psi_{N}^{\Delta}(y)$ to some neighborhood of $\mathcal{Y}_{N}^{\Delta}$ and integrate the system (1) starting from an initial point $y(0) \in \mathcal{Y}_{N}^{\Delta}$ and using $\psi_{N}^{\Delta}(y)$ as a feedback control. The end point of the integration period, $T^{\Delta}$, is identified by the fact that the solution "returns" to a small vicinity of the starting point $y(0)$.

4) Adjust the initial condition and/or control to obtain a periodic admissible pair $\left(u^{\Delta}(\tau), y^{\Delta}(\tau)\right)$ defined on the interval $\left[0, T^{\Delta}\right]$. Calculate the integral $\frac{1}{T^{\Delta}} \int_{0}^{T^{\Delta}} g\left(u^{\Delta}(\tau), y^{\Delta}(\tau)\right) d \tau$ and compare it with $G_{N}^{\Delta}$. If the value of the integral 
proves to be close to $G_{N}^{\Delta}$, then, by (16), the constructed admissible pair is a "good" approximation to the solution of the periodic optimization problem (2).

In conclusion of this section, let us consider the following important special case. Assume that, for all $N$ large and $\Delta$ small enough, the optimal basic solution $\gamma^{N, \Delta}$ of the approximating LPP (15) has the property that

$$
\gamma_{l^{*}, k^{*}}^{N, \Delta}=1, \quad \gamma_{l, k}^{N, \Delta}=0 \quad \forall(l, k) \neq\left(l^{*}, k^{*}\right),
$$

which is equivalent to that the set $\Theta_{N}^{\Delta}$ consists of only one point

$$
\Theta_{N}^{\Delta}=\left\{\left(u_{l^{*}}^{\Delta}, y_{k^{*}}^{\Delta}\right)\right\}
$$

Note that that the indexes $l^{*}, k^{*}$ in (24) and (24) may depend on $N$ and $\Delta$.

Assume that there exists a limit

$$
\lim _{\Delta \rightarrow 0}\left(u_{l^{*}}^{\Delta}, y_{k^{*}}^{\Delta}\right)=(\bar{u}, \bar{y}),
$$

(the same for all sufficiently large $N$ ). Then, as follows from results of [9], the pair $(\bar{u}, \bar{y})$ is the steady state solution of the periodic optimization problem (2) and, in particular,

$$
G_{p e r}=G_{s s}=g(\bar{u}, \bar{y})
$$

\section{Optimal periodic solution for sigmoidal utility function}

In this and the next sections it is always assumed that

$$
Y=\left\{\left(y_{1}, y_{2}\right) \mid y_{i} \in[0,4], i=1,2\right\}
$$

and that $U$ is defined by (7); it is also assumed everywhere that $c=1$ and $B=4$ (see the equations describing the dynamics of the buffer's content (10) and (11)).

Let us consider the interaction between AIMD TCP (8) and the AQM-ECN router (10), the former being taken with $\alpha=1 / 98$ and $\beta=1 / 2$ (such a choice of these parameters corresponds to the case of a single TCP connection and a typical value of the round trip time).

Let us use the objective function (12), with the following values of the parameters: $\kappa=0, M=20$ and $K=5$; and with the sigmoidal utility function being defined by the equation

$$
\psi\left(y_{1}\right)=\frac{y_{1}^{2}}{y_{1}^{2}+a},
$$

with $a=12$. As mentioned in the Introduction section the choice of the sigmoidal utility function correspond to streaming video and audio applications in the Internet (see more details on sigmoidal utility functions in $[6,12,18]$ ). The conventional concave utility functions are analyzed in the next section.

Define the grid of $U \times Y$ by the equations (with $U$ and $Y$ mentioned as above)

$$
u_{i}^{\Delta} \stackrel{\text { def }}{=} i \Delta, \quad y_{1, j}^{\Delta} \stackrel{\text { def }}{=} j \Delta, \quad y_{2, k}^{\Delta} \stackrel{\text { def }}{=} k \Delta .
$$


Here $i=0,1, \ldots, \frac{1}{\Delta}$ and $j, k=0,1, \ldots, \frac{4}{\Delta}$ ( $\Delta$ is chosen in such a way that $\frac{1}{\Delta}$ is an integer). The approximating LPP (15) can be written in this specific case as

$$
G_{N}^{\Delta} \stackrel{\text { def }}{=} \max _{\gamma \in W_{N}^{\Delta}} \sum_{i, j, k}\left(\frac{\left(y_{1, j}^{\Delta}\right)^{2}}{\left(y_{1, j}^{\Delta}\right)^{2}+12}-20 e^{5\left(y_{2, k}-4\right)} y_{1, j}^{\Delta}\right) \gamma_{i, j, k},
$$

where $W_{N}^{\Delta}$ is a polyhedral set defined by the equation

$$
\begin{array}{r}
W_{N}^{\Delta} \stackrel{\text { def }}{=}\left\{\gamma=\left\{\gamma_{i, j, k}\right\} \geq 0: \sum_{i, j, k} \gamma_{i, j, k}=1\right. \\
\left.\sum_{i, j, k}\left(\phi_{i_{1}, i_{2}}^{\prime}\left(y_{1, j}^{\Delta}, y_{2, k}^{\Delta}\right)\right)^{T} f\left(u_{i}^{\Delta}, y_{1, j}^{\Delta}, y_{2, k}^{\Delta}\right) \gamma_{i, j, k}=0,\left(i_{1}, i_{2}\right) \in I_{N}\right\}
\end{array}
$$

in which $\phi_{i_{1}, i_{2}}\left(y_{1}, y_{2}\right) \stackrel{\text { def }}{=} y_{1}^{i_{1}} y_{2}^{i_{2}}$.

The problem (31) was solved using the CPLEX LP solver [20] for $N=5$ and $N=7$ with $\Delta$ varying from 0.00625 to 0.05 . We have obtained the following optimal values of the LPP (31):

$G_{5}^{0.05} \approx 0.07755, G_{5}^{0.025} \approx 0.07757, G_{5}^{0.0125} \approx 0.07757, G_{5}^{0.00625} \approx 0.07758$,

From this data one may conclude that $G_{7}=\lim _{\Delta \rightarrow 0} G_{7}^{\Delta} \approx 0.07756$. Since $G_{7} \geq G_{p e r}$, it follows that, if for some admissible periodic pair $(u(\tau), y(\tau))$,

$$
\frac{1}{T} \int_{0}^{T}\left(\frac{y_{1}^{2}(\tau)}{y_{1}^{2}(\tau)+12}-20 e^{5\left(y_{2}(\tau)-4\right)} y_{1}(\tau)\right) d \tau \approx 0.07756
$$
(2).

then this pair is an approximate solution of the periodic optimization problem Let $\left\{\gamma_{i, j, k}^{N, \Delta}\right\}$ stand for the solution of (31) and define the sets

$$
\begin{aligned}
& \Theta_{N}^{\Delta} \stackrel{\text { def }}{=}\left\{\left(u_{i}, y_{1, j}, y_{2, k}\right): \gamma_{i, j, k}^{N, \Delta} \neq 0\right\}, \\
& \mathcal{Y}_{N}^{\Delta} \stackrel{\text { def }}{=}\left\{\left(y_{1, j}, y_{2, k}\right): \sum_{i} \gamma_{i, j, k}^{N, \Delta} \neq 0\right\} .
\end{aligned}
$$

Let us mark with dots the points on the plane $\left(y_{1}, y_{2}\right)$ which belong to $\mathcal{Y}_{N}^{\Delta}$ for $N=7$ and $\Delta=0.00625$. The result is depicted in Figure 2. The points are represented with $\square$ or $\bullet$ and have an associated $u=1$ or $u=0$, respectively. It is possible to construct a feedback control by using two thresholds for the buffer content. As the queue length $y_{2}$ is decreasing (in the region where $y_{1}<1$ ), we have a certain threshold for when the control should be dropped and allow the data rate $y_{1}$ to grow. The same can be said for the opposite case when the queue 


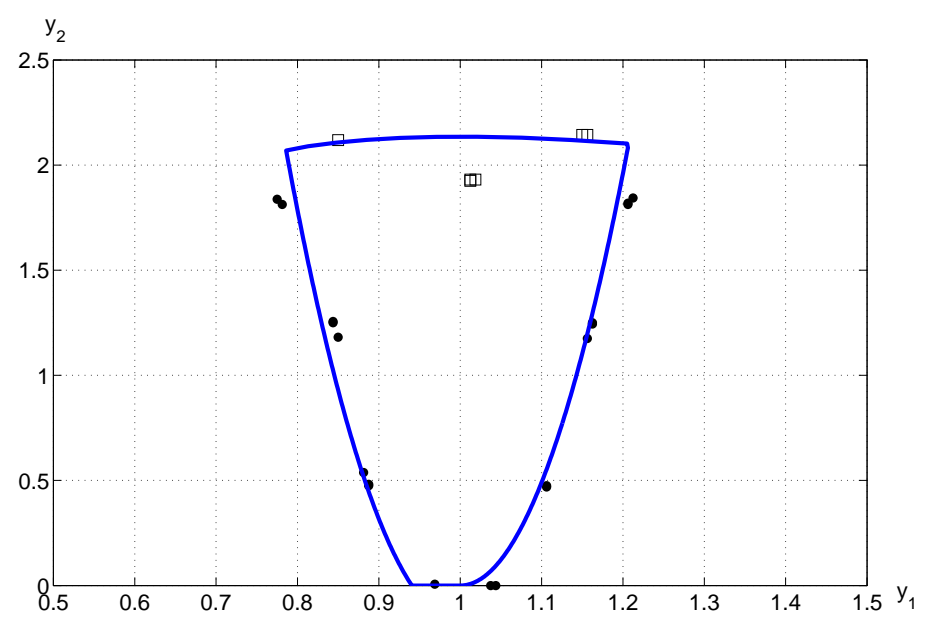

Fig. 2. Optimal state trajectory approximation

is increasing and $y_{1} \geq 1$. The threshold values in our numerical example can be chosen as 2.071 and 2.1, respectively. Thus, the feedback control is defined as

$$
u\left(y_{1}, y_{2}\right)=\left\{\begin{array}{l}
1, y_{1}<1 \text { and } y_{2}>2.071 \\
1, y_{1} \geq 1 \text { and } y_{2}>2.1 \\
0, \text { otherwise }
\end{array}\right.
$$

Using this feedback control, we can integrate the system with the initial point $y_{1}=1, y_{2}=0$. The optimal state trajectory is plotted as a solid line in Figure 2. In Figure 3 we show the evolution of the state variables and the optimal control.

The value of the objective function calculated on this pair is approximately 0.077072. Comparing it with (33), one can conclude that the admissible pair which has been constructed is an approximation to the solution of (2).

Curiously enough, the evolution of the optimal sending rate $y_{1}$ resembles a "saw-tooth" behavior of the "instantaneous" TCP sending rate. We recall that the variables in our fluid model stand for average values. Hence, the optimal solution suggests that the dynamics of the control in AQM should be much slower than the dynamics of TCP. It is also interesting to see that the use of the sigmoidal utility function results in the recommendation to use the control rarely but intensively. In fact, this has a natural explanation. A user of a streaming application prefers to have a good quality of service most of the time and to experience a bad quality of service seldom and for very short periods of time rather than to have a not satisfactory quality of service all the time.

We have also tested the current objective function for the interaction between AIMD and AQM-non-ECN and for the MIMD congestion control. For those cases, we have also detected similar periodic optimal solutions. 

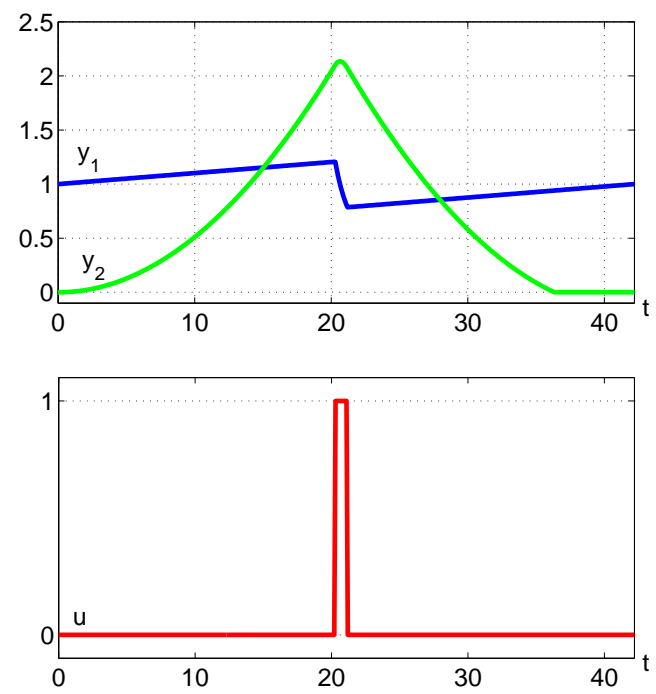

Fig. 3. Approximated periodic solution and optimal control

\section{Optimal steady state solution}

As in the previous section, let us consider the interaction between AIMD TCP (8) and the AQM-ECN router (10). However, in contrast to the above consideration, let us choose the following objective function

$$
g(u, y)=y_{1}-y_{2} .
$$

This choice of the utility function corresponds to the throughput maximization. That is, take $\psi\left(y_{1}\right)=y_{1}, \kappa=1$, and $M=0$ in (12). Note that, as can be easily verified, in this case, the solution of the steady state optimization problem (4) is

$$
\bar{u}=0.02, \quad \bar{y}_{1}=1, \quad \bar{y}_{2}=0
$$

and, in particular, $G_{s s}=\bar{y}_{1}-\bar{y}_{2}=1$. We define the grid of $U \times Y$ as in the previous Section 4.

The approximating LPP (15) in this specific case is of the form

$$
\max _{\gamma \in W_{N}^{\Delta}} \sum_{i, j, k}\left(y_{1, j}^{\Delta}-y_{2, k}^{\Delta}\right) \gamma_{i, j, k}=G_{N}^{\Delta},
$$

where $W_{N}^{\Delta}$ has exactly the same form as in (32).

Proposition 1. For any $N=1,2, \ldots$, and any $\Delta>0$ such that $\frac{0.02}{\Delta} \stackrel{\text { def }}{=} i^{*}$ is integer, there exists a basic optimal solution $\gamma^{N, \Delta} \stackrel{\text { def }}{=}\left\{\gamma_{i, j, k}^{N, \Delta}\right\}$ of the LPP (38) 
defined by the equations

$$
\gamma_{i^{*}, j^{*}, k^{*}}^{N, \Delta}=1, \quad \gamma_{i, j, k}^{N, \Delta}=0 \forall(i, j, k) \neq\left(i^{*}, j^{*}, k^{*}\right),
$$

where $i^{*}$ is as above and $j^{*}=\frac{1}{\Delta}, k^{*}=0$.

Proof of the proposition is given in [2].

Since, by definition, $u_{i^{*}}^{\Delta}=0.02, y_{1, j^{*}}^{\Delta}=1, y_{2, k^{*}}^{\Delta}=0$, that is, $\left(u_{i^{*}}^{\Delta}, y_{1, j^{*}}^{\Delta}, y_{2, k^{*}}^{\Delta}\right)$ coincides with the optimal steady state regime $\left(\bar{u}, \bar{y}_{1}, \bar{y}_{2}\right)$ defined in (37), one obtains the following corollary of Proposition 1 (see (26) and (27)).

Corollary 2. The periodic optimization problem (2) has a steady state solution and this steady state solution is defined by (37). In particular,

$$
G_{p e r}=G_{s s}=\bar{y}_{1}-\bar{y}_{2}=1 \text {. }
$$

We have also checked numerically the other criteria with concave utility functions for the sending rate. It appears that if the utility function for the sending rate is concave, the optimal solution is steady state. The same conclusion holds for the case of interaction between AIMD TCP and AQM-non-ECN and when MIMD is used instead of AIMD.

\section{Conclusions}

We have analyzed the interaction between TCP and AQM using the fluid model approach. The fluid model approach leads to a periodic optimization problem. We have shown that depending on the choice of the utility function for the sending rate, the optimal solution is either periodic or steady state. In particular, we have obtained steady state solution for all concave utility functions and periodic solutions for sigmoidal utility functions. In the case of sigmoidal utility functions, the optimal periodic solution resembles strikingly the "saw-tooth" behavior of the instantaneous TCP sending rate evolution. Moreover, we note that the optimal dynamics of AQM is much slower than the dynamics of TCP. The optimal AQM strategy can be implemented with the help of a simple queue with two thresholds. The choice of the sigmoidal utility functions results in the recommendation to apply the control rarely but intensively. With the help of linear programming approach for periodic optimization we have succeeded to prove that the steady state solution is indeed an optimal solution for the given non-linear periodic optimization problem.

\section{References}

1. K. Avrachenkov, U. Ayesta, and A. Piunovsky, "Optimal Choice of the Buffer Size in the Internet Routers", in Proceedings of IEEE CDC-ECC 2005.

2. K. Avrachenkov, L. Finlay, and V. Gaitsgory, "TCP-AQM Interaction: Periodic Optimization via Linear Programming", in Control Theory Applications in Financial Engineering and Manufacturing, Eds.: H. Yan, G. Yin, and Q. Zhang, Springer (Kluwer), Fred Hillier's International Series in Operations Research and Management Sciences, To Appear. 
3. M. Allman, V. Paxson and W. Stevens, TCP congestion control, RFC 2581, April 1999, available at http://www.ietf.org/rfc/rfc2581.txt.

4. M. Christiansen, K. Jeffay, D. Ott and F. Donelson Smith, "Tuning RED for Web Traffic", IEEE/ACM Trans. on Networking, v.9, no.3, pp.249-264, June 2001. An earlier version appeared in Proc. of ACM SIGCOMM 2000.

5. F. Colonius, "Optimal Periodic Control", Lecture Notes in Mathematics, SpringerVerlag, Berlin, 1988.

6. M. Fazel and M. Chiang, "Network Utility Maximization with Nonconcave Utilities Using Sum-of-Squares Method", in Proceedings of IEEE CDC-ECC 2005.

7. S. Floyd and V. Jacobson, "Random Early Detection Gateways for Congestion Avoidance", IEEE/ACM Trans. on Networking, v.1, no.4, pp.397-413, 1993.

8. V. Gaitsgory, "Suboptimization of Singularly Perturbed Control Problems", SIAM J. Control and Optimization, 30 (1992), No. 5, pp. 1228 - 1240.

9. V. Gaitsgory and S. Rossomakhine "Linear Programming Approach to Deterministic Long Run Average Problems of Optimal Control", SIAM J. Control and Optimization, 44 (2005/2006), No 6, pp 2006-2037.

10. V. Jacobson, Congestion avoidance and control, ACM SIGCOMM'88, August 1988.

11. T. Kelly, "Scalable TCP: Improving performance in highspeed wide area networks", Computer Comm. Review, v.33, no.2, pp.83-91, 2003.

12. J.-W. Lee, R. Mazumdar, and N. Shroff, "Non-Convex Optimization and Rate Control for Multi-Class Services in the Internet", IEEE/ACM Trans. on Networking, v.13, no.4, 2005.

13. S. Low, F. Paganini and J. Doyle, "Internet Congestion Control", IEEE Control Systems Magazine, v.22, no.1, pp.28-43, February 2002.

14. V. Misra, W. Gong and D. Towsley, "A Fluid-based Analysis of a Network of AQM Routers Supporting TCP Flows with an Application to RED", in Proceedings of ACM SIGCOMM 2000.

15. M. May, J. Bolot, C. Diot and B. Lyles, "Reasons Not to Deploy RED", in Proceedings of 7th International Workshop on Quality of Service (IWQoS'99), June 1999, London, UK.

16. J. Postel, User Datagram Protocol, $R F C$ 768, August 1980, available at http://www.ietf.org/rfc/rfc0768.txt.

17. K. Ramakrishnan, S. Floyd and D. Black, The Addition of Explicit Congestion Notification (ECN) to IP, RFC 3168, September 2001, available at http://www. ietf .org/rfc/rfc3168.txt.

18. S. Shenker, "Fundamental Design Issues for the Future Internet", IEEE J. Selected Areas Commun., v.13, no.7, pp.1176-1188, 1995.

19. R. Srikant, The Mathematics of Internet Congestion Control, Birkhaüser, Boston, 2004.

20. ILOG CPLEX http://ilog.com/products/cplex/ 